\title{
Merleau-Ponty, Beaufret, Heidegger : Parménide ou la découverte de l'ontologie
}

\section{Franck Robert}

\section{(2) OpenEdition}

1 Journals

Édition électronique

URL : http://journals.openedition.org/alter/1731

DOI : 10.4000/alter.1731

ISSN : 2558-7927

Éditeur :

Association ALTER, Archives Husserl (CNRS-UMR 8547)

\section{Édition imprimée}

Date de publication : 1 octobre 2010

Pagination : 277-295

ISBN : 2-9522374-6-8

ISSN : $1249-8947$

\section{Référence électronique}

Franck Robert, « Merleau-Ponty, Beaufret, Heidegger : Parménide ou la découverte de l'ontologie », Alter [En ligne], 18 | 2010, mis en ligne le 01 juin 2020, consulté le 27 juin 2020. URL : http:// journals.openedition.org/alter/1731; DOI : https://doi.org/10.4000/alter.1731 


\section{MERLEAU-PONTY, BEAUFRET, HEIDEGGER : PARMÉNIDE OU LA DÉCOUVERTE DE L'ONTOLOGIE ${ }^{1}$}

Franck Robert

Indéniablement, le projet philosophique du Visible et l'invisible et des recherches ultimes de Merleau-Ponty a un sens ontologique; tout aussi indéniable est le fait que Merleau-Ponty, dans les dernières années de sa vie, devient un lecteur attentif de Heidegger, non seulement de Sein und Zeit bien sûr, mais également du second Heidegger, du Heidegger d'après le tournant ${ }^{2}$. Les notes de cours au Collège de France de l'année 1958-1959, dans le cours intitulé La Philosophie aujourd'hui, l'attestent: Merleau-Ponty, après des cours consacrés à Husserl envisage "la philosophie comme problème » chez Heidegger ${ }^{3}$. L'on sait moins en revanche que c'est une méditation sur la dialectique qui a suscité cet intérêt de Merleau-Ponty pour le second Heidegger. C'est dans cette même méditation, dans le cours de 1955-1956, que la pensée de Merleau-Ponty prend un tour décisivement ontologique. Dialectique, ontologie et découverte de la méditation philosophique du second Heidegger relèvent d'un seul et même mouvement de pensée chez Merleau-Ponty. C'est une telle hypothèse que nous nous proposons d'examiner ici.

En 1955-1956, Merleau-Ponty consacre son cours au Collège de France à la dialectique, mais la perspective de ce cours n'est plus aucunement celle

1. Le texte ici proposé reprend une conférence présentée le 27 mars 2010 à la troisième Journée d'étude consacrée aux inédits de Merleau-Ponty et organisée par Emmanuel de Saint Aubert à l'École normale supérieure de la rue d'Ulm, journée intitulée «Relire Merleau-Ponty à partir des inédits ». Je tiens à remercier infiniment Emmanuel de Saint Aubert tout à la fois pour ses précieuses recherches personnelles sur l'ensemble de l'œuvre de Merleau-Ponty et pour être le mettre d'œuvre de ces journées d'études collectives autour des inédits.

2. Sur la lecture merleau-pontyenne de Heidegger, mais également de Husserl, et pour une première étude de la lecture merleau-pontyenne de Parménide, voir notre livre, Phénoménologie et ontologie, Merleau-Ponty lecteur de Husserl et Heidegger, Paris, L'Harmattan, 2005.

3. Notes de cours, 1959-1961, texte établi par Stéphanie Ménasé, Paris, Gallimard, 1996, p. 91-148. 


\section{L'attention}

des Aventures de la dialectique: le projet ne relève pas d'une pensée du politique et de l'histoire seulement, le projet est explicitement d'ordre ontologique. Merleau-Ponty recherche dans ce cours le sens de l'ontologie moderne, ontologie qui est tout aussi bien sa propre ontologie, ontologie de l'être à distance, de l'être comme transcendance. Une telle ontologie ne semble cependant pouvoir se penser que comme dialectique. Elle interdit en effet de penser l'être comme positif, comme identité : le négatif, la différence, la contradiction, l'écart sont inhérents à l'Être même. Seule la dialectique semblerait pouvoir dire et penser cela. En retour, la dialectique serait aussi ce qui pourrait se comprendre à partir de l'ontologie moderne. La circularité est ici féconde : les «descriptions de l'être sensible, l'être du monde, l'être d'univers", écrit Merleau-Ponty dans les notes de cours ${ }^{4}$, permettent $\mathrm{d}^{\prime}$ « illustrer [la] dialectique comme vie commune des contradictoires et leur médiation ${ }^{5}$ ", mais la pensée de la dialectique est en retour pensée de ce qui a pu se donner sur un mode intuitif dans les descriptions de la chose, du monde, de l'univers.

L'ontologie moderne est ainsi pour Merleau-Ponty « la renaissance de la dialectique $^{6} »$. Dire cela, $c^{\prime}$ est dire non seulement qu'il y a eu une naissance et une histoire de la dialectique, mais c'est dire aussi que cette histoire a bien pu perdre le sens initial de la dialectique, son sens essentiel. Merleau-Ponty écrit même que l'ontologie moderne retrouverait le «plus pur de la dialectique $^{7}$ ", qualification de pureté plutôt inhabituelle chez MerleauPonty. Que peut bien être en effet ce sens le plus pur ? Le trouvera-t-on dans l'origine et l'histoire de la dialectique elle-même, dans sa naissance ? On s'attendrait à voir en Platon l'acte de naissance de la dialectique, mais il faudrait plutôt le trouver selon Merleau-Ponty chez les Présocratiques, chez Parménide et Héraclite. Dans le cours, le commentaire de Merleau-Ponty porte cependant essentiellement sur Le Poème de Parménide. C'est après avoir analysé la pensée de Zénon à partir du Parménide de Platon et de Bergson que Merleau-Ponty se consacre à la lecture de Parménide. Vient de paraître, en novembre 1955, la traduction et l'introduction que Jean Beaufret propose du Poème $e^{8}$. Merleau-Ponty y découvre une lecture heideggérienne de Parménide: c'est elle qui guide l'ensemble de son commentaire. Fera

4. Les notes de cours sont conservées à la Bibliothèque Nationale de France, rue Richelieu. Le cours de 1955-1956 correspond au volume XIV des inédits, intitulé, décrit et référencé : Volume XIV. Collège de France. 1955-1956. Cours du jeudi. La philosophie dialectique et cours du lundi. Textes et commentaires sur la dialectique. 255 ff. MF 13249 (R130149). Pour les références, nous noterons : Vol. XIV, pagination BNF des microfilms, suivie de la pagination de Merleau-Ponty.

5. Vol. XIV, 183, II 13.

6. Vol. XIV, 184, II 14.

7. Vol. XIV, 184, II 14.

8. Parménide, Le Poème, traduction et présentation par Jean Beaufret, Paris, PUF, 1955. 
suite à ce commentaire une lecture plus précise de Platon, du Parménide en particulier à partir de l'étude qu'en donne Jean Wahl en $1926^{9}$.

Rappeler la démarche de ce cours n'est pas seulement une information indiquant la chronologie du cours de Merleau-Ponty, c'est aussi préciser que la naissance philosophique de la dialectique est affaire complexe, dont la forme est elle-même dialectique ${ }^{10}$. Non seulement la naissance de la dialectique est elle-même complexe, puisqu'elle relève de reconstructions, d'interprétations, voire de légendes, comme le note David Bélot dans une étude consacrée à ce même cours ${ }^{11}$, mais c'est l'idée même d'une naissance de la dialectique qui mérite d'être interrogée : on se demandera ainsi ce que signifie une telle naissance, comment la considérer, quel statut lui accorder. Dire les choses ainsi, c'est évidemment souligner d'emblée qu'en ce cours sur la dialectique se noue un dialogue nouveau pour Merleau-Ponty avec Parménide aux matins de la philosophie et Heidegger son contemporain.

Que la rencontre avec ces interlocuteurs se fasse à partir de la question de la dialectique peut cependant surprendre. Parménide serait plutôt le penseur de l'Être, de la plénitude ontologique que du processus dialectique et $\mathrm{du}$ négatif. Heidegger rejetterait quant à lui la dialectique du côté de la métaphysique de la subjectivité qui aurait oublié la question de l'Être. On ne comprendra dès lors cette importance de Parménide et, de manière indirecte, de Heidegger, que si l'on élucide le rapport essentiel de la dialectique à l'ontologie. Ce qui revient pour nous à répondre à deux questions : 1) En quel sens Merleau-Ponty peut-il chercher le sens d'une ontologie nouvelle dans une pensée de la dialectique? 2) Comment peut-il découvrir en Parménide et les présocratiques le sens initial de la dialectique, dans cette pensée qui est d'abord naissance peut-être de l'ontologie ? Pour répondre à ces questions, nous suivrons les grands moments du cours que MerleauPonty consacre à Parménide.

\section{Interprétation de Parménide}

Merleau-Ponty souligne d'abord l'originalité de l'interprétation que Beaufret et Heidegger donnent de Parménide. Il faut d'emblée voir dans l'opposition entre une interprétation «classique» et cette nouvelle interprétation l'opposition entre une ontologie positive qui absolutise l'être

9. Jean Wahl, Etude sur le Parménide de Platon, Paris, F. Rieder et Cie, 1926 (réédition Paris, Vrin, 1951).

10. Voir Vol. XIV, 175, II 5.

11. David Bélot, « Dialectique, ontologie et histoire dans les notes préparatoires aux cours sur La philosophie dialectique (1956) », Revue Internationale de Philosophie, $n^{\circ} 62$, p. 197. L'auteur cite le volume XIV, p. 173. Je tiens particulièrement à remercier David Bélot, qui prépare la transcription et l'édition de l'ensemble de ce cours et qui m'a permis de confronter ma propre transcription d'extraits à l'ensemble de son travail. 


\title{
L'attention
}

et le néant en soulignant leur opposition radicale et l'ontologie que MerleauPonty cherche alors à penser, ontologie de la latéralité, de l'être à distance.

Mais en ce cas, pourrait-on objecter d'emblée, Parménide n'est-il pas celui qui précisément pense l'Être et le néant comme absolus? Tel pourrait bien être en effet le sens du Fragment II du Poème:

\begin{abstract}
Eh bien donc je vais parler - toi, écoute mes paroles et retiens-les - je vais te dire quelles sont les deux seules voies de recherche à concevoir : la première - comment il est et qu'il n'est pas possible qu'il ne soit pas - est le chemin auquel se fier-car il suit la Vérité. La seconde, à savoir qu'il n'est pas et que le non-être est nécessaire, cette voie, je te le dis, n'est qu'un sentier où ne se trouve absolument rien à quoi se fier. Car on ne peut ni connaître ce qui n'est pas - il n'y a pas là d'issue possible -, ni l'énoncer en une parole ${ }^{12}$.
\end{abstract}

Le premier chemin, «chant de la vérité », est le chemin de l'Être. Le second chemin, impraticable, est le chemin du néant. On voit dès lors mal comment l'on pourrait trouver là une pensée de la dialectique et de l'Être à distance. Merleau-Ponty résume ainsi l'interprétation classique du Poème :

Interprétation habituelle: l'être est, le néant n'est pas - négation du multiple, de l'autre, de la non-identité, du temps (éternité) - ens realissimum ${ }^{13}$.

L'attention de Merleau-Ponty ne se porte donc pas sur cette opposition radicale entre l'être et le néant, entre deux chemins dont un seul, celui de l'être, serait praticable. S'arrêter dans notre lecture à ces deux seules voies, ce serait lire Parménide comme penseur de l'identité, alors que ce que cherche Merleau-Ponty est une pensée du mélange, de la médiation. Précisément, la lecture que proposent Heidegger et Beaufret ne s'arrête pas là. Beaufret, à partir de Heidegger ${ }^{14}$, met en évidence l'importance de la troisième voie, celle des dokounta, des choses en tant qu'elles apparaissent, des apparences, voie qui serait celle de l'opinion. Une telle voie est habituellement discréditée. Parménide semble lui-même la rejeter comme voie de l'errance et de l'erreur, voie où apparaît «ce qu'ont en vue les mortels, où l'on ne peut se fier à rien de vrai 15 ». Merleau-Ponty le rappelle à son tour : le sentier que suivent les « double-têtes », hommes non philosophes, égarés, errants, est «labyrinthe ${ }^{16} »$, palintropos. S'engager dans la troisième

12. Parménide, op. cit., p. 79.

13. Vol. XIV, 185, III 1.

14. Beaufret cite (op. cit., p. 27) la note qui guide toute sa lecture de Parménide : «K. Reinhardt a pour la première fois [...] conçu et résolu le problème si souvent débattu des deux parties du poème philosophique de Parménide, bien qu'il ne montre pas expressément le fondement ontologique que présuppose la connexion de $l^{\prime} \dot{\alpha} \lambda \hat{\eta} \theta \varepsilon \varepsilon \alpha$ et de la $\delta$ ó $\xi \alpha$, ainsi que la nécessité de ce fondement. » (traduction de Beaufret, op. cit., 27, Sein und Zeit, p. 223).

15. Parménide, op.cit., p. 79, Fragment I, 30.

16. Ibid., p. 81. 
voie semble dès lors «peu compatible avec [la] rigueur des descriptions de la $1^{\text {re }}$ voie $^{17}$.»

$C^{\prime}$ est pour cette raison que les interprétations classiques ont disqualifié cette troisième voie. Beaufret le rappelle dans l'article qu'il consacre à Parménide dans les Philosophes célèbres, volume dirigé par Merleau-Ponty : il n'y aurait "pas de place entre l'être et le non-être pour une troisième possibilité ${ }^{18}$. » Deux interprétations s'opposent alors pour conduire au même discrédit. Cette voie serait celle de prédécesseurs, Héraclite ou Pythagore, qu'il faudrait réfuter, voie fausse de ceux qui sont dans l'erreur. Ou bien cette voie serait la voie de l'hypothèse, de ceux qui ne parviendraient pas à penser «le paradoxe de l'opposition philosophiquement exhaustive de l'être et du non-être ${ }^{19}$ ». L'une et l'autre interprétations rejetteraient la troisième voie du côté du faux et de l'irréalité.

Mais on comprend mal alors l'unité du Poème qui ne parle pas seulement de l'essentiel - l'Être de la première voie - mais qui donne également à voir ce qui selon la troisième voie se déploie, comme le dit la fin du Fragment VIII :

Le déploiement de ce qui paraît, en tant qu'il se produit comme il se doit, voilà ce
que je vais te révéler en entier, afin que le sens des mortels jamais ne te dépasse 20

L'essentiel est moins en effet dans l'opposition des deux voies, écrit Beaufret, que «dans l'unité indivisible du Poème, intimement liée au contraste de la vérité de l'être et de la non-vérité du non-être», unité qui oblige à porter toute son attention à la non-vérité du non-néant de la troisième voie $^{21}$. Dès lors, s'il s'agit bien de la voie de l'errance ou de l'erreur, il faut s'y engager cependant, il ne faut pas s'arrêter à la seule critique de ce qu'ont en vue les mortels. Merleau-Ponty qui, depuis La Structure du Comportement et plus encore la Phénoménologie de la perception, ne cesse d'interroger le va-et-vient ambigu et nécessaire de l'attitude naturelle et de l'attitude transcendantale, de l'irréfléchi et de la réflexion, du Lebenswelt et de la pensée philosophique, ne peut qu'être sensible à l'exhortation du Poème :

Or, il faut que tu sois instruit de tout, du cour sans tremblement de la vérité, sphère accomplie, mais aussi de ce qu'ont en vue les mortels, où l'on ne peut se fier à rien de vrai. Mais oui, apprends aussi comment la diversité qui fait montre d'elle-

17. Vol. XIV, 185, III 1.

18. Jean Beaufret, article « Parménide » dans Les Philosophes célèbres, sous la direction de Maurice Merleau-Ponty, Ed. Citadelles et Mazenod, 1956, réédition sous le titre Les Philosophes de l'Antiquité au XXe siècle, histoire et portraits, Paris, Librairie générale française, 2006, p. 134.

19. Ibid., p. 134.

20. Parménide, op.cit., p. 89, Fragment VIII, 60, cité en partie par Merleau-Ponty en Vol. XIV, 185, III 1.

21. Beaufret, op.cit., p. 134. 


\section{L'attention}

même devait déployer une présence digne d'être reçue, étendant son règne à travers toutes choses 22 .

La troisième voie est donc une voie recevable ${ }^{23}$. Une telle voie est praticable même si elle est voie d'hommes non philosophes, dikranoi ${ }^{24}$, « doubletêtes », " pour qui l'être et aussi bien le non-être, le même et ce qui n'est pas le même, font $\operatorname{loi}^{25}$ ", écrit Parménide. Selon une logique de l'identité, cette double affirmation serait contradictoire, ces hommes sont donc égarés. Mais «il y a une vérité de $1^{\prime}$ opinion $^{26}$ », précise Merleau-Ponty. Cette vérité, n'estce pas la vérité même de l'apparaître en son déploiement? Avec les dokounta de Parménide, écrit Beaufret,

le monde de l'illusion n'est pas encore pour Parménide une illusion de monde,
mais, dans son éclat et dans sa gloire, la présence même des choses de ce monde-ci
au lieu unique et central de leur manifestation ${ }^{27}$.

Pour qu'une telle lecture de Parménide soit possible, note Merleau-Ponty,

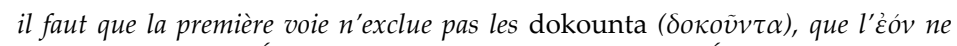
soit pas un Suprême Étant, mais ce dont participent tous les Étants, ce qui fait que les $\hat{E}$ tres sont $\hat{E}$ tres - que le chemin ne soit pas vers un transcendant ${ }^{28}$.

Une compréhension onto-théologique de l'ontologie interdirait de penser cette réhabilitation des dokounta. C'est tout à la fois une lecture platonicienne et chrétienne de Parménide qui conduirait à un rejet de la troisième voie. Les dokounta, simples apparences pour Platon, seraient à écarter et le regard devrait se porter vers les Idées, seules permanentes et pleinement réelles. Mais une autre lecture de Parménide est possible, qui refuse toute pétition de platonisme. La réhabilitation des dokounta, telle que Merleau-Ponty l'effectue à partir de Beaufret et Heidegger, est possible si Platon, loin de représenter un progrès par rapport à Parménide, ouvre les voies d'une certaines décadence: Platon en pensant l'Être comme transcendant pourrait bien représenter un recul par rapport à une pensée ontologique authentiquement dialectique. Loin d'exclure la non-vérité de la vérité, loin de se réfugier dans l'arrière-monde des Idées, il faudrait assumer le mélange de la vérité et de la non-vérité, de l'être et du non-être.

La voie des dokounta oblige ainsi à refuser une opposition radicale entre l'être de la première voie et le néant de la seconde :

22. Parménide, op. cit., p. 79, Fragment I, 28-32.

23. Vol. XIV, 185, III 1.

24. Parménide, op.cit., p. 81, Fragment VI, 5.

25. Ibid.

26. Vol. XIV, 185, III 1.

27. Beaufret, op.cit., p. 136.

28. Vol. XIV, 185, III. 
[La troisième voie], écrit Beaufret, n'est pas la voie de l'être, mais pas davantage celle du non-être. Elle est la voie d'un non-néant ou d'une non-vérité qui est cependant viable comme la voie de la vérité ou de l'être et non pas inviable comme la non-vérité du non-être 29

Il y a donc lieu de ne pas négliger ce que peut signifier la voie des dokounta, en laquelle nous avons notre site.

La troisième voie ne nous enferme pas dans l'aveuglement de ceux qui ne voient pas ou dans la surdité de ceux qui n'entendent pas, cette voie nous oblige à découvrir l'« étrangeté de l'étant lui-même 30 », étrangeté qui conduit à penser la différence de l'être et de l'étant. L'interprétation de Beaufret, écrit Merleau-Ponty,

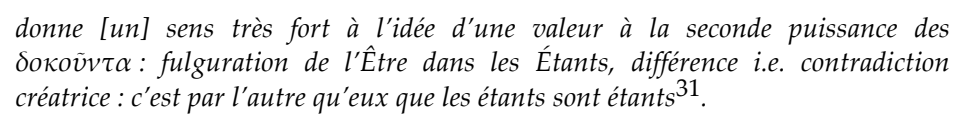

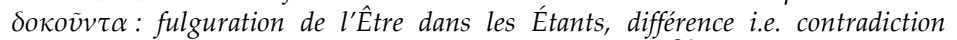
créatrice: c'est par l'autre qu'eux que les étants sont étants 31.

De manière ramassée, se lisent là les enjeux même de la pensée de Merleau-Ponty, enjeux qui peuvent se dire dorénavant en termes ontologiques. Ce que découvre ici Merleau-Ponty, c'est ainsi la différence ontologique heideggérienne. La différence entre la première et la troisième voie dévoile la différence entre l'Être et l'étant: les étants ne sont ce qu'ils sont que par leur participation à l'Être; en différant de l'Être tout en y participant, ils inscrivent le non-être en l'Être. Cette différence nomme peutêtre ce que Merleau-Ponty ne cesse de chercher dans une perspective phénoménologique déjà ancienne: comment l'apparaître se produit-il? Comment se joue à travers les apparitions multiples de ce qui est l'apparaître même?

Soulignons cependant que Merleau-Ponty pense cela à la fois en termes ontologiques et dialectiques: remarquons en effet son insistance, non heideggérienne ici, sur le processus et la dialectique, sur ce qui se nomme ici la «différence », c'est-à-dire la «contradiction créatrice » entre l'Être et les étants.

Comment dès lors penser l'Être auquel les étants participent ? À quelle conception de l'Être sommes-nous alors conduits? N'assiste-on pas à un coup de force de Beaufret et de Heidegger qui voudraient voir ici la différence ontologique de l'Être et de l'étant?

Merleau-Ponty souligne d'abord ce que Beaufret néglige dans son commentaire : il y néglige en premier lieu la pensée parménidienne de l'Être comme plénitude. Certes Beaufret néglige cela, mais c'est précisément pour contester une lecture seulement partielle du Poème. Merleau-Ponty de même contesterait une compréhension positive de l'Être à laquelle pourrait inviter

29. Beaufret, op.cit., p. 134.

30. Vol. XIV, 185, III 1.

31. Vol. XIV, 188, III 4. 


\section{L'attention}

Le Poème de Parménide. Plutôt que comme sphère, c'est comme ouverture qu'il faut penser l'Être.

Le second point que néglige Beaufret est le caractère atemporel et immuable de l'Être: Parménide pense l'Être comme «immobile, éternel, sans croissance ${ }^{32} »$. Mais Merleau-Ponty n'accepte pas une telle approche de l'Être. Dans sa lecture ultérieure de Heidegger, il pensera l'Être comme physis; avec Whitehead, il pensera la nature comme passage, l'Être comme processus. C'est alors vers Héraclite tel qu'on le comprend habituellement qu'il faudrait se tourner. La fin des cours consacrés à la dialectique chez Parménide et Platon envisage d'ailleurs l'étude d'Héraclite. Merleau-Ponty y insiste sur le lien du processus et de la dialectique :

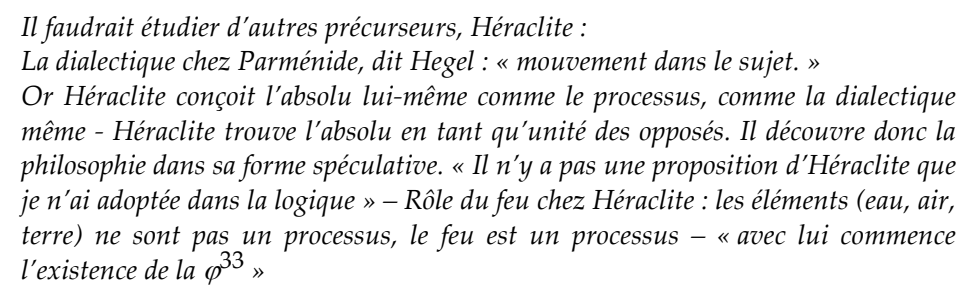
je n'ai adoptée dans la logique »-Rôle du feu chez Héraclite : les éléments (eau, air, terre) ne sont pas un processus, le feu est un processus - "avec lui commence l'existence de la $\varphi^{33}$ »

$\mathrm{Au}$ risque qui consisterait à s'enfermer dans la plénitude de l'Être parménidien sans croissance, il faudrait opposer l'exigence héraclitéenne d'une pensée du processus.

Enfin, Beaufret néglige l'opposition radicale de l'être et du non-être qui semble se dégager du Poème. L'un ne surgit pas de l'autre. Les deux chemins, le premier et le second, sont incompatibles et totalement distincts pour Parménide. Ce serait donc seulement dans un retour au chemin de l'être, par passage par la troisième voie, que l'on trouverait un être « rongé de néant ${ }^{34}$ ».

Ce que semble manquer ici Beaufret, c'est précisément ce que MerleauPonty pourrait critiquer chez Parménide si l'on réduisait Parménide à la pensée des deux seuls chemins de l'être et du non-être. Ce que Beaufret néglige, c'est ce que Merleau-Ponty critiquera ultérieurement lorsqu'il refusera de penser l'être comme plénitude et le néant comme absolu. Cependant, tout ce que Beaufret laisse de côté dans son commentaire ne rend que plus décisif, aux yeux de Merleau-Ponty, ce qu'il met en évidence. Ce qui importe, c'est comment se déploie la différence de l'Être et de l'étant, comment l'Être de la première voie peut ne pas exclure les dokounta.

Il ne les exclut pas parce que l'Être doit être pensé, souligne MerleauPonty, «comme membrure ${ }^{35}$ ». Le terme, pour qualifier $l^{\prime} \hat{E}$ tre, peut

32. Vol. XIV, 188, III 4.

33. Vol. XIV, 211, 27 février.

34. Vol. XIV, 188, III 4.

35. Vol. XIV, 185, III 1. 
surprendre. Peut-être avons-nous là, dans le Poème de Parménide, l'une des origines de ce mot si décisif dans l'ontologie ultime de Merleau-Ponty. Le terme est utilisé au Fragment VIII, lorsque Le Poème décrit les caractères de l'Être : inengendré, impérissable, de membrure intacte, inébranlable et sans fin, tout entier, un, d'un seul tenant, sans génération ${ }^{36}$. La membrure semble pouvoir dire ainsi pour Merleau-Ponty la manière qu'ont les étants de participer à l'Être. La membrure serait la manière qu'a l'Être d'imprégner touts les étants qui sont, de les faire tenir ensemble et eux-mêmes comme étants.

S'il est certes difficile de dire que s'annonce ici une compréhension de l'Être comme Être sensible, cela peut néanmoins éclairer le sens que donnera ultérieurement Merleau-Ponty à l'idée de membrure : le mot est peut-être là pour dire en termes sensibles la différence ontologique heideggérienne. Dans Le Visible et l'invisible, la membrure est ce qui, invisible, soutient tout le visible, elle est ce néant qui fait le sens du visible et en constitue l'armature. À titre d'exemple, citons une note de travail de novembre 1959 qui, parmi d'autres notes, conserve la mémoire de ce cours sur la dialectique :

Le sens est invisible, mais l'invisible n'est pas le contradictoire du visible: le visible a lui-même une membrure d'invisible, et l'in-visible est la contrepartie secrète du visible, il ne paraît qu'en lui, il est le Nichturpräsentierbar qui m'est présenté comme tel dans le monde - on ne peut l'y voir et tout effort pour l'y voir, le fait disparaître, mais il est dans la ligne du visible, il en est le foyer virtuel, il s'inscrit en lui (en filigrane) ${ }^{37}$.

La membrure invisible du visible semble dire ainsi de manière sensible la membrure de l'Être qui assemble les étants.

Ce qui, dans le cours sur la dialectique, quelques années avant la rédaction des notes du Visible et l'invisible, permet alors de penser ce lien entre l'être à la membrure intacte et les étants, c'est l'idée de participation. La membrure de l'être auquel les étants participent conduit ainsi à penser la différence ontologique en termes de participation. L' le Poème ne renvoie pas à tel étant singulier mais à l'Être auquel tous les étants participent. Il importe de bien entendre ce qui se dit dans le participe eon. Beaufret, à la suite de Heidegger dans Qu'appelle-t-on penser?, insiste ainsi sur la double dimension du participe grec, double dimension dont le eon comme participe du verbe einai, être, est un cas particulier non seulement exemplaire mais décisif. Le participe fait à la fois signe vers le substantif et le verbe. L'eon dit ainsi l'étant et le fait d'être de tout étant. Il dit la participation de chaque étant à l'Être. Dans le participe se dit la différence ontologique :

36. Parménide, op.cit., p. 83, Fragment. VIII,

37. Le Visible et l'invisible, suivi de Notes de travail, texte transcrit par Claude Lefort, Paris, Gallimard, 1964 (noté VI.), p. 269. 


\section{L'attention}

Cette Différence qui distingue et tient écartés l'un de l'autre l'être et l'étant, mais pour les unir l'un et l'autre en une éclosion originelle, écrit Beaufret, nous pouvons maintenant reconnaître en elle la singulière relation que protège encore, dans le langage, l'incomparable ambiguité du participe. Une telle relation, plus initiale que toute causalité émanant de l'étant est, dans la transgression de tout étant simplement donné, la relation même de transcendance dans laquelle ce qui vient au jour, à travers l'oscillation entre l'être et le non-être, c'est la dimension même de l'être de l'étant ${ }^{38}$.

Dans la différence du visible et de l'invisible, différence sensible, peutêtre est-ce bien d'une telle participation dont il s'agit : nous trouvons là, avec les idées de membrure, de différence, d'oscillation entre l'être et le non-être, de dimension, quelques-uns des termes essentiels à l'ontologie ultime de Merleau-Ponty. Mais au moment du cours sur la dialectique, avec l'idée de participation, Merleau-Ponty est conduit à une relecture de Platon. Penser le rapport de l'Être et des étants en termes de différence et de participation oblige en effet à une critique nécessaire de l'idée d'essences séparées chez Platon. Refuser de lire Parménide à partir de Platon, c'est donc aussi se demander s'il ne faut pas également relire Platon sans l'enfermer dans le réalisme des Idées.

\section{Interprétation de Platon}

Suite à cette lecture du Poème, on serait tenté d'opposer Parménide à Platon ${ }^{39}$, aux penseurs, comme les nomme Nietzsche ${ }^{40}$, Hinterweltler, penseurs des arrière-mondes. Merleau-Ponty le rappelle, Beaufret met bien en garde les lecteurs de Parménide : il faut éviter toute pétition de platonisme, et toute critique hâtive de la troisième voie, voie des mortels, voie de l'opinion. Merleau-Ponty cependant nuance cette approche en s'obligeant à relire autrement Platon, notamment à partir du Parménide, du Sophiste et du Théétète, non plus seulement à partir de La République.

Une relecture de Platon s'impose en effet : si un discrédit de l'apparence, de la doxa, peut se trouver chez Platon, ce serait dans les textes de $L a$ République, ceux dont parle Beaufret d'abord. On pourrait trouver en revanche chez Platon lui-même cette troisième voie dont parle Parménide qui interdirait toute disqualification philosophique de la doxa; on pourrait ainsi retrouver chez Platon une pensée du "dimorphisme de l'étant ${ }^{41}$ », de l'étant aux formes multiples, changeantes, une pensée même de la différence de l'Être et de l'étant, de la différence ontologique :

38. Beaufret, Introduction au Poème de Parménide, op.cit., p. 45-46.

39. Vol. XIV, 189, III 5.

40. Beaufret, op.cit., p. 32

41. Vol. XIV, 189, III 5. 


\begin{abstract}
La $\delta o ́ \xi \alpha$ n'est-elle qu'une apparence, note Merleau-Ponty, domaine intermédiaire, qui doit le céder à la science, si les mythes (ici-bas, au-delà, réminiscence) ne sont pas expression au niveau de l'opinion d'un mystère philosophique qui ne serait pas très différent $d u$ "dimorphisme de l'étant». [...] Comment dire que le Bien de Platon tend à être un étant alors que Platon le met manifestement au-delà de l'être ${ }^{42}$ ?
\end{abstract}

Trois idées méritent donc d'être examinées chez Platon : 1) l'ouverture de la doxa à la vérité, le rapport non-vérité, vérité, 2) l'idée d'un foisonnement des étants, dont les formes changeantes ne doivent pas être disqualifiées au profit de la permanence des Idées, 3) et l'idée, enfin, que le Bien n'a rien d'un Étant, qu'il ne saurait être pensé comme Étant suprême.

Le mouvement de lecture de Merleau-Ponty est donc singulier: il faut non seulement relire Parménide sans pétition de platonisme, dans l'esprit de Beaufret, mais il faudrait même relire Platon, sans cette même pétition de platonisme, à partir de cette relecture initiale de Parménide. Il faudrait relire Platon, pourrait-on dire, sans pétition de parricide ni pétition de christianisme. C'est à cela que se consacrera la suite du cours du lundi sur la dialectique. C'est ainsi en un mouvement lui-même dialectique qu'il faudrait relire Platon, mouvement dialectique qui engage, à partir de cette double relecture nécessaire, de Parménide et de Platon, une réinterprétation du sens de la dialectique.

\title{
3. Interprétation de la dialectique
}

Merleau-Ponty confronte ces interprétations à ce qu'il nomme la pensée par arguments et la pensée subjective, ainsi qu'à la dialectique hégélienne.

Il analyse d'abord la pensée par arguments. Pourrait être contestée chez Platon, chez Zénon d'abord, la pensée par arguments qui conduit dans les dialogues les interlocuteurs à se contredire. Une telle pensée ne saurait être féconde, elle serait elle-même prise dans les contradictions qu'elle dénonce, pensée "ventriloque», capable de dire et penser une chose et son contraire. Merleau-Ponty n'accepte pas une telle interprétation de Platon : il faut plutôt voir dans la pensée de Platon une manière de mettre en jeu des contraires, d'intégrer la contrariété et le mouvement même de la dialectique dans le processus de la pensée. Il n'y aurait pas ainsi une décadence platonicienne par rapport à Parménide.

Merleau-Ponty considère ensuite la "pensée subjectiviste». Les questions de Platon, du Théétète notamment sur le savoir et le non-savoir, la pensée du faux, n'enferment pas dans le subjectivisme. Leur sens porte tout à la fois sur la vérité et sur l'être :

42. Vol. XIV, 189, III 5. 


\section{L'attention}

Ces questions ne nous enferment pas dans la subjectivité, note Merleau-Ponty, puisque Platon veut les deux: comprendre l'accès à l'être et la possibilité de l'erreur. - Elles existent comme questions : la question d'à $\lambda \dot{\eta} \theta \varepsilon \iota \alpha^{43}$.

Merleau-Ponty voit dans ce passage par la pensée subjective une nécessité pour la dépasser précisément: l'accès à l'Être, à l'Être comme ouverture, passe par une réflexion sur le faux et l'erreur. Un tel accès ne saurait être direct. En cela, Merleau-Ponty critique Heidegger et son refus de penser la subjectivité comme moment même de la vérité :

\footnotetext{
Est-ce simple décadence ?, demande Merleau-Ponty. Non, c'est expérience à faire. Pour dépasser la subjectivité, il faut l'avoir connue. Le surgissement de l'être sur fond de néant (Heidegger) serait-il possible sans cette connaissance ${ }^{44}$ ?
}

Ce passage par la subjectivité exigerait que la vérité pensée comme aletheia soit aussi pensée à partir du sujet, sans qu'il s'agisse pour autant d'adosser cette vérité et l'Être au sujet, comme chez Descartes, Kant ou Hegel. Passer par le sujet est nécessaire pour dépasser le subjectivisme ensuite.

Parménide est enfin brièvement confronté à la pensée hégélienne de la dialectique. Merleau-Ponty insiste moins sur la totalisation que sur le mouvement même de la dialectique. Un tel mouvement de retour à soi et de sortie de soi est présent chez Hegel même, comme le rappelle fréquemment Merleau-Ponty. Que la totalisation soit à l'horizon de toute pensée fait certes partie du mouvement dialectique, mais cela ne signifie pas que ce mouvement doive se résorber dans une totalisation ultime :

\footnotetext{
D'ailleurs certaine totalisation faite d'avance est impliquée ds toute philosophie, c'est, de la dialectique, un élément valable: chez Parménide lui-même:

[...] « Ce m'est tout un par où je commence, car là même à nouveau je viendrai en retour 45 ».

L'ouverture à l'être, si elle est primordiale, est ouverture au tout ${ }^{46}$.
}

La dialectique pense ainsi l'ouverture à l'Être comme «ouverture au tout ${ }^{47}$ ", mais les deux demeurent essentiels: le tout et l'ouverture. Privilégier l'un serait annuler l'autre ; ce serait annuler la dialectique ellemême. La vraie dialectique doit maintenir l'ouverture, mais il n'y aurait pas d'ouverture à l'Être s'il n'y avait pas d'ouverture au tout, à titre d'horizon. C'est un tel mouvement que l'hyperdialectique, au moment du Visible et l'Invisible, cherchera à penser :

43. Vol. XIV, 190, III 6.

44. Vol. XIV, 190, III 6.

45. Parménide, op.cit., p. 81, Fragment V.

46. Vol. XIV, 190, III 6.

47. Vol. XIV, 190, III 6 


\begin{abstract}
La mauvaise dialectique commence presque avec la dialectique, et il n'est de bonne dialectique que celle qui se critique elle-même et se dépasse comme énoncé séparé; il n'est de bonne dialectique que l'hyperdialectique [...] Ce que nous appelons hyperdialectique est une pensée qui [...] est capable de vérité, parce qu'elle envisage sans restriction la pluralité des rapports et ce qu'on a appelé l'ambiguité. [...] Ce que nous rejetons ou nions, ce n'est pas l'idée du dépassement qui rassemble, c'est l'idée qu'il aboutisse à un nouveau positif, à une nouvelle position ${ }^{48}$.
\end{abstract}

Si l'hyperdialectique n'est pas nommée encore, elle s'offre déjà cependant comme un possible de la philosophie. La méditation sur le Poème de Parménide indique dès lors le sens que Merleau-Ponty cherche à donner à sa philosophie: réfléchir sur la dialectique, c'est envisager un nouveau philosopher.

\title{
4. La philosophie
}

L'on peut voir dans le cours consacré à Parménide la manière qu'a Merleau-Ponty d'envisager la philosophie dans cette dernière moitié des années 1950. Ce cours ouvre notamment sur sa découverte critique du second Heidegger et sur l'approfondissement ontologique de sa pensée. Il constitue un moment décisif dans l'orientation ontologique de sa pensée.

La question initiale du cours sur la dialectique est d'abord celle du commencement en philosophie. Dès l'élaboration du cours sur la dialectique, cette question se pose à Merleau-Ponty ${ }^{49}$. L'examen des formes historiques de la dialectique, de Platon à Marx, doit permettre de découvrir le sens même de la dialectique, sans que ce sens ne soit déjà établi et préjugé :

\begin{abstract}
Nous ne préjugeons pas qu'il y a une philosophie dialectique dont l'un et l'autre relèvent: ce sera justement l'objet du cours de l'établir, de cerner cette $\varphi$ dialectique immanente à tous, qui les juge tous, et dont ils ont révélé des côtés, et qu'il faut poursuivre. Tout ce qu'on peut exiger est que cette hypothèse soit précisée, que son existence ne soit pas vaguement présupposée. Bref il faut bien commencer, et il y a qqs présupposés qu'on doit seulement ne pas masquer, pour les mettre en question ${ }^{50}$.
\end{abstract}

La question du commencement met en jeu ce qui se joue de manière décisive dans la dialectique : c'est du présupposé dont il faut partir si l'on veut accéder à la vérité, c'est du non-vrai sans doute qu'il faut partir pour accéder au vrai. Dans le commencement est en outre engagé le sens même de la l'histoire de la philosophie : on ne se demandera pas seulement «par quoi commencer?», mais également "quelle peut-être la valeur d'un commencement? ?, et notamment «quel sens historique peut-il avoir dans

48. VI. 129.

49. Vol. XIV, 5, I 1.

50. Vol. XIV, 5, I 1. 


\section{L'attention}

l'histoire de la dialectique et pour l'histoire de la philosophie? » Si la question de la dialectique et de son histoire a aussi un sens ontologique, on pourra se demander quel est le sens ontologique de ce commencement, s'il a un sens ontologique particulier. Si ce commencement est Parménide, on ne pourra éviter une confrontation avec Heidegger et sa méditation sur le sens à donner au commencement présocratique de la philosophie. Ce n'est pas alors seulement la question du commencement qui se pose, c'est aussi celle de l'histoire de la philosophie elle-même.

À la question du commencement en philosophie, et du sens de ce commencement, est liée en effet la pensée de l'histoire de la philosophie, de son rapport à la philosophie elle-même. Ce rapport n'est pas d'abord soumis, pour Merleau-Ponty, à l'histoire de l'Être au sens heideggérien du terme, c'est seulement ultérieurement, après une lecture effective du second Heidegger, qu'il affrontera la question d'une Seinsgeschichte, dans les notes de travail et dans le cours de 1958-1959 sur La Philosophie aujourd'hui. Dès ce cours cependant, Merleau-Ponty est conduit, à partir de Parménide, Beaufret et Heidegger, vers des contrées nouvelles pour lui, là où l'histoire de la philosophie prend un sens ontologique. Merleau-Ponty rencontre pour la première fois dans le Poème et dans le commentaire de Beaufret l'idée de Moira, idée tout à la fois d'un destin de l'Être et de la pensée, d'une pensée à dessein de l'Être :

La pensée destinée à l'être, non constituante de l'être, Moĩ $\alpha$ qui fait l'histoire, qui est la futurition dans l'origine - Tel serait le sens $d u: c^{\prime} e s t$ une seule chose penser et ce à dessein de quoi il y a pensée ${ }^{51}$.

On sait l'importance de l'idée de Moira, divinité du partage et du destin, pour Heidegger dans la conférence du même nom et dans Qu'appelle-t-on penser ?. Cette importance implique chez Heidegger, chez Beaufret lecteur de Heidegger, puis chez Merleau-Ponty dans ce commentaire, une méditation sur le sens originaire de la philosophie. Un tel sens, qui pourrait bien demeurer obscur, mérite interprétation. Une telle interprétation, note Merleau-Ponty, pourrait bien emprunter aux Pré-socratiques eux-mêmes les termes qui permettraient de penser l'origine et le devenir de la philosophie $^{52}$. Derrière la lecture par Beaufret et Heidegger de Parménide, de Platon et de la dialectique, l'on pourrait trouver « un Philosophieren qui se cache, qui « fait signe » à travers les présocratiques ${ }^{53}$ ».

Dire cela ne signifie cependant pas revenir à des significations établies chez Parménide. Ce serait en résorber le sens-énigme. Revenir aux Présocratiques, pour Merleau-Ponty comme pour Heidegger, ce n'est pas réduire la philosophie à l'élucidation ou au maniement de significations instituées, de concepts établis. À une telle philosophie, il faut opposer une

51. Vol. XIV, 185, III 1.

52. Vol. XIV, 191, III 7.

53. Vol. XIV, 191, III 7. 
philosophie qui conçoive la parole comme «instauration d'un monde ${ }^{54}$ », ouverture, parole qui peut en un sens aussi peut-être maintenir l'énigme de ce qu'elle pense. Ce serait bien là l'une des choses qui se jouent dans le Poème de Parménide. Être ainsi attentif à l'origine même du penser philosophique, c'est aussi refuser une philosophie qui chercherait dans le terme d'une histoire un sens vrai, le sens absolu de cette histoire. La critique merleau-pontyenne vise un «Hegel écrasant dans sa pensée tout le passé55. » Mais Merleau-Ponty n'accepte pas davantage une philosophie qui chercherait dans l'origine un sens vrai, sens pur et originel. Il refuse la posture de Heidegger à l'égard des Grecs, il refuse Heidegger tel que Beaufret le comprend: si la pensée selon Heidegger signifie un retour à une origine pure, Heidegger ne serait qu'un symétrique inversé de Hegel.

Pour Merleau-Ponty, la lecture de Parménide ou d'Héraclite ne nous renvoie aucunement en effet à une origine oubliée, aube à retrouver de la pensée occidentale. Il y a, entre Merleau-Ponty et la lecture de Parménide par Beaufret et Heidegger une vraie divergence quant à la pensée du commencement et quant au statut à accorder à la pensée grecque. Cette lecture en effet, si riche et féconde soit-elle, relève d'une interprétation, qui peut être contestable:

\begin{abstract}
Textes de Parménide sont très lacunaires et ne portent que difficilement tout cela. On est obligé d'interpréter : volonté du philosophe de se replier dans une Parole d'avant qui résonne jusqu'à lui et en lui. La philosophie ici se définit en histoire de la philosophie - Cela n'est pas défendu: [En marge: mais c'est acte philosophique.] on peut analyser, lire Parménide librement, méditer sur lui. Cela ne serait défendu que si la philosophie voulait s'imposer comme histoire, tacitement, au lieu de se présenter comme philosophie. Ceci est à mettre au compte de l'acte philosophique de Heidegger plutôt que de Parménide 56 .
\end{abstract}

La lecture relève donc d'une interprétation, elle nous dit peut-être davantage de la pensée de Heidegger que de celle de Parménide. Ce qui, évidemment, du point de vue heideggérien, est contestable, si dans le Poème de Parménide se dit ou doit s'entendre quelque chose de fondamental pour la pensée elle-même, en dehors de la lecture que, lui, Heidegger, peut en faire.

Cette situation lacunaire des textes de Parménide est par ailleurs essentielle non seulement pour ce qui est de la question de l'interprétation, mais également pour ce qui est du rapport pensée langage : de telles lacunes sont accidentelles en partie bien sûr, mais elles sont aussi peut-être propres au texte lui-même qui, du point de vue de la pensée conceptuelle, est décisivement lacunaire. Il s'agit précisément d'un poème, et c'est comme tel qu'il nous faut précisément le lire.

54. Vol. XIV, 191, III 7.

55. Vol. XIV, 191, III 7.

56. Vol. XIV, 9. 


\title{
L'attention
}

Se pose donc la question de la manière que nous avons de lire aujourd'hui Parménide, de la manière que nous avons d'écouter sa parole. L'écoute d'une telle parole serait pour Heidegger l'écoute d'une Parole d'avant, qui vient jusqu'à lui, et qui résonne en lui, mais que nous ne parvenons pas à entendre. Rien de tel pour Merleau-Ponty, qui refuse toute nostalgie qui ferait résider dans le passé, un passé originel, le sens même de la pensée, de l'histoire, de l'être ou de la philosophie :

\begin{abstract}
Donc droit d'une réflexion sur les vues de l'être des philosophes, réflexion immanente, par Einfühlung et non inductive, à condition qu'elle ne se donne pas pour recours à un Paradis perdu, à un secret, à une clef, à un sens propre déposé dans le passé [Ajouté: ou quelque part au monde] et d'avance comme acte philosophique, comme "explication » avec le passé ${ }^{57}$.
\end{abstract}

Sans doute tout cela est-il plus complexe chez Heidegger, sans doute Beaufret accentue-t-il l'idée d'une origine perdue de la pensée grecque, mais reste que Merleau-Ponty ne partage pas l'idée d'une aube oubliée et d'un destin ontologique de la pensée occidentale. Pourtant, tout en se démarquant fermement de cette thèse heideggérienne, il sera lui-même conduit à penser l'histoire en termes ontologiques et l'être en son historicité.

L'ensemble du cours sur la dialectique le suggère, mais la méditation sur le concept d'institution dans les années 1950, notamment dans le cours de 1954-1955, y invite déjà. Avec Parménide, c'est bien à l'institution de la philosophie que nous avons affaire. La puissance philosophique de Parménide ne tient pas pour Merleau-Ponty à son caractère ésotérique et originel, mais à son caractère fondateur et à sa présence contemporaine dans la pensée. C'est ainsi en termes d'institution, de Stiftung, qu'il faudrait penser ce rapport que nous entretenons avec les Grecs, non en termes d'héritage seulement ou de retour nécessaire à une parole pure et originaire. C'est du moins ce que précise l'article de présentation des philosophes grecs que Merleau-Ponty rédige pour l'ouvrage collectif consacré aux Philosophes célèbres, article précisément intitulé Les Fondateurs: si certains peuples ont rencontré la philosophie, « les Grecs l'ont instituée ${ }^{58}$ ». Leur pensée nous est contemporaine, familière même :

Quand nous reprenons leurs textes, après vingt-cinq siècles, c'est notre vie même qui palpite entre leurs mots, notre manière d'interroger le monde. Il y a là un langage qui se traduit terme à terme, des mouvements, des reprises de la pensée, des silences qui sont nôtres. Nous sommes de plain-pied ${ }^{59}$.

On pourrait certes dire, concède Merleau-Ponty, que cela n'a rien d'étonnant, puisque nous sommes les héritiers des Grecs. Mais ce n'est pas

57. Vol. XIV, 9.

58. Merleau-Ponty, op.cit., p. 121.

59. Ibid. 
là un simple héritage dont il s'agit, puisque le geste par lequel nous reprenons les Grecs ne relève pas seulement d'une influence dont on aurait hérité ; ce geste est celui d'une reprise créatrice d'un acte créateur initial. Dans les termes de Husserl et des cours sur l'institution, ou du cours sur L'Origine de la géométrie, l'on parlera d'une Nachtstiftung :

L'extraordinaire est justement que ce qu'ils [les Grecs] ont trouvé garde une puissance de suscitation intacte. Que les philosophes, à travers les siècles, s'identifient dans ce qu'ils ont de meilleur au Grecs, cela même est au crédit des Grecs et les qualifie comme fondateurs 60 .

La fondation originaire est ainsi ouverture d'un horizon :

\begin{abstract}
Ces acquisitions [de questions philosophiques que les Grecs n'ont pas posées en tant que telles mais qui se trouvent déjà en leur pensée] demeurent dans l'horizon qu'ils ont ouvert parce qu'ils ont créé et compris le genre de question qui fait la philosophie $^{61}$.
\end{abstract}

Si les Grecs sont en un sens nos contemporains, c'est parce que nous sommes aussi capables d'un acte de reprise à partir de l'horizon qu'ils ont ouvert: l'Urstiftung n'est telle que si elle est reprise dans la Nachstiftung qu'elle appelle et a rendu possible.

Dans ce cours sur la dialectique, lorsque la parole grecque est envisagée en son caractère initial et initiateur, cela ne signifie pas que l'on doive se replier dans cette parole des premiers temps de la philosophie. Une telle parole ne peut parler encore que si nous la faisons parler, que si nous maintenons par notre propre parole sa puissance créatrice. Le faire philosophique ne saurait donc signifier ni une fixation dans une pensée ultime et totalisante, ni le repli dans une parole originaire. En ce sens, si pour Merleau-Ponty comme pour Heidegger la Parole nous a, c'est aussi nous qui avons la parole. Si le passé est à ce point fondamental, c'est qu'il nous est contemporain, nous vivons "un échange entre le passé et le présent $^{62}$ ». Tel est le sens même de la dialectique, qui peut bien être aussi le projet philosophique d'un nouveau philosopher, projet de Merleau-Ponty en cette seconde moitié des années 1950 :

C'est à notre sens l'indication d'une tâche de la dialectique vraie : faire comprendre l'affinité des époques et des moments du temps, sans les réduire à un "retour", à un progrès "inévitable », sans détruire leur caractère de créations 63 .

60. Ibid.

61. Ibid., p. 122.

62. Vol. XIV, 191, III 7.

63. Vol. XIV, 191, III 7. 


\section{L'attention}

\section{Conclusion}

S'il faut voir avec Beaufret dans le Poème de Parménide le « Poème de la méthode $^{64}$ » qui serait le socle fondateur de toute méthode philosophique, sans doute faut-il dire que Merleau-Ponty ne cesse de s'inspirer de cette méthode, en privilégiant cependant cette voie que Parménide semblerait pouvoir écarter, la voie des dokounta, des étants tels qu'ils viennent à la présence. Si la voie de l'Être est certes viable pour Parménide, elle pourrait bien être impraticable pour Merleau-Ponty si elle n'était pas doublée de cette troisième voie qui en l'Être préserve le négatif, l'absence, le non-être. Seule la troisième voie peut être l'occasion tout à la fois d'une ontologie et d'une dialectique.

D'une ontologie, parce qu'en elle se déploie la différence de l'Être et de l'étant, d'un Être pensé comme Élément en lequel tout étant participe, comme membrure maintenant ensemble les étants. Cette différence que la troisième voie des dokounta fait apparaître manifeste aussi l'inscription du non-être dans l'Être : si la seconde voie du néant est impraticable, ce n'est pas tant parce que nul néant ne serait, mais c'est parce que affirmer le néant seul et absolu c'est interdire tout cheminement et toute pensée ; il en va tout autrement si le néant est incorporé à l'être, si le néant est ce qui maintient l'écart de tout étant avec l'Être dont il participe, si le néant qui dans les dokounta imprègne et conditionne toute présence est indissociable de tout apparaître ${ }^{65}$.

C'est ainsi à partir de cette troisième voie que l'on trouve le cœur de la dialectique qui renaît avec l'ontologie moderne. Mais de quelle dialectique s'agit-il ici ? Il y a dialectique d'abord parce qu'avec cette troisième voie s'ouvre le champ de l'aletheia, champ en lequel se joue le rapport de la vérité et de la non-vérité, champ en lequel dans l'errance des mortels empruntant cette voie se dévoile l'étant en son apparaître, errance que le penseur doit observer pour ouvrir la pensée à la vérité. Il y a dialectique ensuite parce que se découvre alors l'Élément en lequel les contradictoires peuvent se distinguer et s'unir, cet Élément est l'Être qui en lui maintient le non-être.

A-t-on bien là la pureté annoncée de la dialectique? Ce n'est pas sûr, si cette pureté est elle-même à chercher: on a néanmoins le socle d'une ontologie à découvrir encore - une ontologie qui dès lors se confrontera plus directement à la pensée du second Heidegger, sans adopter jamais son refus de la dialectique. Ce qu'une telle ontologie doit pouvoir penser, c'est tout à la fois l'articulation de la vérité et de la non-vérité, l'ouverture à l'Être, une histoire de l'Être peut-être, une différence ontologique au cœur du sensible, mais la pensée de cela devra maintenir la différence interne à l'Être, l'ouverture sans totalisation, sans clôture de la pensée sur elle-même et sur un tout auquel elle accéderait. Seule une hyperdialectique, dialectique sans

64. Beaufret, op.cit., p. 133.

65. Voir Vol. XIV, 13. 
totalisation pourrait, comme le suggère Le Visible et l'invisible, lier ainsi la pensée de la dialectique et la pensée ontologique de l'écart, de la différence, du Sensible.

Le cours de 1955-1956, et plus particulièrement la lecture du Poème de Parménide, nous semble être, dans le cheminement de Merleau-Ponty, l'événement d'une telle pensée en émergence. 